\title{
Chromosome dynamics in bacteria: triggering replication at opposite location and segregation in opposite direction
}

\author{
Ady B. Meléndez ${ }^{\dagger}$, Inoka P. Menikpurage ${ }^{\dagger}$, and Paola E. Mera*
}

Department of Chemistry and Biochemistry, New Mexico State University, Las Cruces, NM 88003, USA.

* Correspondence: Paola E. Mera, Department of Chemistry and Biochemistry, CB W380, 1175 North Horseshoe Drive, Las Cruces, NM 88003-8001.

mera@nmsu.edu

\section{$\dagger$ Equal contributors}

Key words: Chromosome replication, chromosome segregation, DnaA, ParA, centromere, Caulobacter crescentus 


\begin{abstract}
The accurate onset of chromosome replication and segregation are fundamental for the survival of the cell. In bacteria, regulation of chromosome replication lies primarily at the initiation step. The bacterial replication initiator DnaA recognizes the origin of replication (ori) and opens this double stranded site allowing for the assembly of the DNA replication machinery. Following the onset of replication initiation, the partitioning protein ParA triggers the onset of chromosome segregation by direct interactions with ParB-bound to the centromere. The subcellular organization of ori and centromere are maintained after the completion of each cell cycle. It remains unclear what triggers the onset of these key chromosome regulators DnaA and ParA. One potential scenario is that the microenvironment of where the onset of replication and segregation take place hosts the regulators that trigger the activity of DnaA and ParA. In order to address this, we analyzed whether the activity of DnaA and ParA are restricted to only one site within the cell. In non-dividing cells of the alpha proteobacterium Caulobacter crescentus, ori and centromere are found near the stalked pole. To test DnaA's ability to initiate replication away from the stalked pole, we engineered a strain where movement of ori was induced in the absence of chromosome replication. Our data show that DnaA can initiate replication of the chromosome independently of the subcellular localization of ori.

Furthermore, we discovered that the partitioning protein ParA was functional and could segregate the replicated centromere in the opposite direction from the new pole toward the stalked pole. We showed that the organization of the ParA gradient can be completely reconstructed in the opposite orientation by rearranging the location of the centromere. Our data reveal the high flexibility of the machineries that trigger the onset of chromosome replication and segregation in bacteria. Our work also provides insights into the coordination between replication and segregation with the cellular organization of specific chromosomal loci.
\end{abstract}




\section{INTRODUCTION}

Triggering the onset of chromosome replication at the correct frequency and under the right conditions is central to the survival and proliferation of the cell. In bacteria, DnaA is the highly conserved initiator of chromosome replication [1]. DnaA is an AAA+ protein that recognizes a specific chromosomal locus known as the origin of replication (ori) [2, 3]. DnaA binds ori forming a helical right-handed polymeric structure, which exerts a torsional stress that results in the opening of the DNA strands [4, 5]. Once DnaA opens the double stranded chromosome, the replication machinery assembles at ori and initiates chromosome replication bidirectionally.

The multiple levels of DnaA's regulation reveal the significant investment the cell makes to accurately initiate chromosome replication. DnaA's ability to bind ori can be regulated by either ori-binding proteins that outcompete DnaA for binding sites, or by non-ori chromosomal loci that can sequester DnaA away from ori $[6,7]$. Proteins like DiaA (E. coli) and HobA (H. pylori) facilitate DnaA's ability to form a helical polymeric structure at ori by recruiting multiple DnaA molecules [8-10]. Furthermore, the ability of DnaA to open the DNA strands can be turned off by the induction of its ATPase activity. Only DnaA bound to ATP can form the polymeric structures at ori required for strand opening [11]. DnaA's activity can be turned off by HdaA, which stimulates DnaA-ATP hydrolysis [12-14]. In vitro analyses of DnaA's interaction with membrane phospholipids have also been shown to induce the dissociation of the nucleotide bound and thus potentially regulate DnaA's activity $[15,16]$. In $C$. crescentus, the levels of DnaA in the cell can also be cell-cycle regulated [17-20].

The subcellular location where DnaA initiates chromosome replication is established by the position of ori inside the cell. Depending on the bacterial species, the subcellular location of ori varies significantly. For instance, C. crescentus ori is found at one pole (the stalked pole), whereas in E. coli and $B$. subtilis, ori is found near mid-cell. However, within a single species, the subcellular location of ori is strictly retained at the same position in non-dividing cells and re-established soon after chromosome replication and segregation initiate in actively dividing cells. In non-dividing cells of $C$. crescentus, ori is retained near the stalked pole by the interaction between the anchoring protein PopZ and the centromere (parS) [21-23]. Thus, DnaA initiates chromosome replication at ori near the stalked pole and terminates replication at ter locus near the opposite pole (referred here as the new pole) (Fig. 1).

Once chromosome replication initiates at ori, the replication fork must pass through the centromere (parS, 8 kilobases away from ori) for chromosome segregation to initiate [24]. The centromere is bound by a multi-protein complex that plays essential roles in the cell cycle [21-25]. For example, the chromosome is anchored at one pole by interactions between the protein ParB and PopZ (a polar anchoring protein) [21-23] (Fig. 1). Soon after replication initiates, one of the two ParB-coated centromeres is segregated to the new pole by direct interactions between ParB and the ATPase ParA [24, 26-29]. ParA forms a stable gradient with concentrations gradually decreasing from the new pole to the stalked pole. The establishment of this ParA gradient is required for transport directionality [30]. ParB induces ATP-hydrolysis of ParA, releasing ParA from this gradient. Notably, ParA's stable gradient is known to be established well before chromosome replication and segregation are initiated [26-28]. Thus, the mechanism that triggers ParA to initiate segregation of one centromere to the opposite pole remains unclear.

What regulates the timing for DnaA to initiate replication with such precise periodicity remains unsolved. What regulates the timing for ParA to initiate segregation only after the replication of the 
centromere is completed also remains unsolved. One potential scenario is that the microenvironment of the stalked pole in C. crescentus, where the onset of replication and segregation take place, hosts the regulators that trigger the activity of DnaA and ParA. With the goal to further understand what regulates the onset of these key cell cycle regulators (DnaA and ParA), we examined whether the onset of replication and segregation are restricted to ori-centromere's intrinsic localization, which in $C$. crescentus is near the stalked pole. To resolve this question, we genetically engineered a $C$. crescentus strain where movement of ori/centromere can be triggered in the absence of replication initiation [31]. Once ori was translocated to different sites over the cell, we tested for competency of replication initiation and chromosome segregation. Our data revealed that DnaA and ParA are capable of initiating chromosome replication and segregation irrespective of the cellular location of ori and centromere. We show that the organization of the ParA gradient is completely flipped in orientation once the un-replicated centromere localized at the new pole. Our results uncover the robustness and flexibility of the chromosome replication and segregation machineries in bacteria.

\section{RESULTS}

Construction of indicator strain with fluorescently labeled origin of replication

To determine the cellular localization of ori, we engineered a fluorescent tag to be inserted near ori using the Yersinia pestis parS(pMT1) chromosomal sequence and its corresponding gene-encoding ParB(pMT1) fluorescently tagged [32]. The parS-ParB(pMT1) system from Yersinia has been previously used in $C$. crescentus and shown not to interfere with the activity of the native $C$. crescentus ParABS partitioning system [24]. Consistent with those findings, our analyses of growth curves and Colony Forming Units (CFU) revealed that strains with the parS(pMT1) insertion near ori and the expression of $\operatorname{parB}(\mathrm{pMT} 1)$ have no significant effect on the doubling time and viability compared to wild-type (Fig. S1. A \& B). To control the expression of $d n a A$, we first engineered an additional copy of $d n a A$ to replace the gene vanA resulting in the expression of $d n a A$ regulated by the VanA promoter (vanA::dnaA) [33]. Using this merodiploid strain, the native gene encoding for DnaA was replaced with a spectinomycin cassette leaving no scars on the genome. The final indicator strain with a fluorescent label near ori and encoding a single copy of $d n a A$ (regulated by $\mathrm{P}_{\text {van }}$ ) is referred here as PM500 with the genotype $x y I X:: p a r B(p M T 1)$, parS(pMT1) at nucleotide 1,108, dnaA:: $\Omega$, vanA::dnaA. For simplification from here on, we will refer to the parS(pMT1) localized near ori simply as ori.

\section{Sub-physiological levels of DnaA results in translocation of ori away from stalked pole}

In C. crescentus, the centromere is the first chromosomal locus to segregate away from the stalked pole [24]. Previous analyses of cells expressing sub-physiological levels of DnaA (not sufficient to initiate replication) revealed a DnaA-dependent and replication-independent segregation of the centromere [31]. Under these sub-physiological levels of DnaA, cells move the un-replicated centromere from the stalked pole to the new pole. Based on those results, we asked if the same subphysiological levels of DnaA could also trigger the movement of ori, independently of replication. To reach sub-physiological levels of DnaA in the cell, we used the same vanillate promoter to regulate $d n a A$ expression [31]. Using our indicator strain PM500, we tracked the localization of ori using highresolution microscopy. Our results show that sub-physiological levels of DnaA trigger the translocation of ori away from the stalked pole independently of chromosome replication (Fig. 2A). As the time of DnaA depletion increased, cells displayed un-replicated ori focus progressively moving toward the new pole (Fig. 2B). Within $3 \mathrm{~h}$ of DnaA depletion, $\sim 75 \%$ of cells exhibited their ori localized at the new pole. These results resemble the replication-independent movement of the centromere 
suggesting that ori and centromere translocate in a DnaA-dependent manner likely due to their close proximity on the chromosome [31].

DnaA's ability to initiate replication is not restricted to the stalked pole

In C. crescentus, DnaA initiates replication only once per cell cycle and only in stalked cells, which have their ori localized at the stalked pole (Fig. 1). To determine whether DnaA can initiate replication outside the stalked pole, we analyzed cells that had undergone replication-independent translocation of ori. To track replication initiation, we supplemented vanillate to cells with ori localized outside the stalked pole and followed the appearance of two ori foci (Fig. 3A). Thirty minutes after the addition of vanillate, $\sim 60 \%$ of cells with ori localized at the new pole had initiated chromosome replication as evidenced by two clearly separated ori foci. Quantification of the frequencies of replication initiation based on location of ori after $3 \mathrm{~h}$ DnaA depletion revealed that replication initiation was triggered slightly quicker, yet statistically significant, from ori localized at the new pole compared to those localized at the stalked pole (Fig. 3B). Consistent with these results, a similar pattern in rates of replication initiation based on location of ori were observed when tracking the location of a chromosomal locus found 8 kilobases away from ori (Fig. S2). In sum, our results revealed that DnaA's activity as a replication initiator is not restricted to the stalked pole.

\section{Centromeres are effectively segregated in the opposite direction}

Based on our results that DnaA is able to initiate replication from the new pole (Fig. 3), we then formulated our next question concerning chromosome segregation. Can the partitioning system ParABS initiate segregation of the centromere from the new pole toward the stalked pole, which in this case would be in the opposite direction? To test this, we used a $C$. crescentus strain in which the native parB gene was replaced with the fusion gene encoding CFP-ParB and in which the only copy of $d n a A$ was regulated under the vanillate promoter (PM109) [31]. In C. crescentus, the partitioning protein ParB binds directly to the centromere [24, 34]. Thus, we can track centromere movement by using cells expressing a functional fusion protein CFP-ParB. PM109 cells grown in the presence of vanillate were shown to display wild-type dynamics for CFP-ParB localization [31]. When the inducer vanillate is removed from the growth media of PM109, cells are exposed to sub-physiological levels of DnaA insufficient to initiate chromosome replication [31]. Fluorescent imaging of PM109 cells grown without vanillate revealed that the localization of a single CFP-ParB focus with the following distribution: $\sim 37 \%$ at or near the new pole, $\sim 54 \%$ at or near the stalked pole, and $\sim 10 \%$ at/around mid-cell (Fig. S3) These percent distribution of un-replicated centromere localization triggered in a replication-independent manner are consistent with previous analyzes [31].

To determine the ability of ParABS to trigger segregation of the centromere in the opposite direction, we tracked the movement of CFP-ParB in PM109 cells with translocated un-replicated centromeres supplemented with vanillate (Fig. 4). Similar to our analyses of ori, two clearly separated centromeres were observed soon after $d n a A$ expression was reestablished, irrespective of the initial localization of centromere prior to $d n a A$ induction. Notably, cells with two CFP-ParB foci were able to also segregate their centromeres to the cell poles, irrespective of the initial localization of centromere prior to $d n a A$ induction (Fig. 4A). Quantification of these data revealed that the rate at which centromeres were segregated to the cell poles were significantly faster in cells with centromeres that departed from the mid-cell (Fig. 4 B \& C). Chromosome segregation that initiated from either the stalked pole or the new pole was not statistically different. These data suggest that the partitioning protein ParA was able to reorganize its gradient in order to segregate centromeres from mid-cell or from the new pole. 
Active ParA is required for centromere segregation in the opposite direction.

C. crescentus cells expressing ParA variants that are unable to hydrolyze ATP cannot segregate their centromere to the cell poles [24, 26, 35]. To determine whether the segregation of centromere segregation observed from the new pole/mid-cell is ParA-dependent, we tracked CFP-ParB localization in a merodiploid strain that encodes the wild-type allele of parA at the native locus and a dominant negative mutant parA allele regulated by the xylose-inducible promoter. This dominant negative allele encodes a missense mutation in the ATPase domain (ParA ${ }^{\text {D4AA }}$ ) of ParA that inhibits chromosome segregation [36]. To test for segregation in the opposite direction, we first allowed cells to translocate their un-replicated centromeres by growing them in growth media devoid of vanillate. Xylose was then supplemented to the growth media $1 \mathrm{~h}$ prior to the addition of vanillate so that replication initiation was induced in the presence of the dominant negative ParA ${ }^{\mathrm{D} 44 \mathrm{~A}}$. Our data revealed that $>80 \%$ of cells expressing wild type ParA were able to segregate the centromeres to the poles, as evidenced by one CFP-ParB focus at each pole (Fig. 5). However, cells expressing the dominant negative ParA $^{\mathrm{D} 44 \mathrm{~A}}$ after replication initiated outside the stalked pole failed to segregate their replicated centromeres as evidenced by two CFP-ParB near each other (Fig. 5 A \& B). These data strongly suggest that the segregation observed of the centromere in the opposite direction from the new pole to the stalked pole requires an active chromosome segregation machinery.

\section{Re-localization of the centromere locus triggers rearrangement of ParA gradient}

In wild-type C. crescentus, ParA forms a gradient with high concentrations at the new pole that gradually decrease toward the stalked pole (Fig. 1) [26-28]. Our observation that the centromere could be segregated in the opposite direction suggested that cells with centromeres at the new pole rearranged the gradient of ParA. To determine whether ParA could change the orientation of its gradient, we assessed the localization patterns of ParA using the background of a parA merodiploid strain that contains the wild-type allele of parA at the native locus and a fluorescently-tagged parA (ParA-mCherry) under the inducible promoter for xylose [36]. This construct grown under normal DnaA expression conditions, in the presence of vanillate, displayed wild type localization dynamics of ParA-mCherry. However, cells with un-replicated centromeres that had translocated to the new pole displayed a completely flipped pattern of the ParA-mCherry gradient (high levels at the stalked pole that gradually decrease toward the new pole) (Fig. 6A). Our data revealed that similarly to DnaA and replication initiation, ParA can rearrange to initiate the segregation of the centromere from outside the stalked pole.

\section{PopZ bi-polar localization is triggered independently of chromosome replication}

The directionality of ParA's chromosome segregation from the stalked pole to the new pole has been proposed to be dependent on the localization of the anchoring protein PopZ [36]. To determine whether the onset of chromosome replication/segregation from outside the stalked pole altered PopZ localization dynamics, we tracked the localization of PopZ by using cells expressing a functional fusion protein mCherry-PopZ. In our control experiment with cells grown in the presence of vanillate (the $d n a A$ inducer), mCherry-PopZ foci localized one at each pole upon the onset of chromosome replication and segregation [21-23, 31]. In cells with translocated un-replicated centromeres, mCherryPopZ localization was dependent on the localization of CFP-ParB (Fig. 6B). $90 \%$ of cells with CFPParB at the stalked pole displayed a single mCherry-PopZ focus also localized at the stalked pole. $\sim 90 \%$ of cells with CFP-ParB localized at the new pole displayed two PopZ-CFP foci with one localized at each pole. Notably, cells with CFP-ParB localized at mid-cell displayed an equal 
combination of cells with either one mCherry-PopZ focus localized at the stalked pole or two PopZCFP foci localized one at each pole. Upon the induction of chromosome replication by the addition of the inducer vanillate, cells with bi-polar localization of PopZ seemed remained bi-polar (Fig. 6B). Regardless where the centromere was localized when replication initiation was induced by vanillate supplementation, $\sim 100 \%$ of cells displayed bi-polar localization of mCherry-PopZ (Fig. 6C).

Effects in viability from initiating chromosome replication/segregation from outside the stalked pole.

To determine whether initiating chromosome replication and/or segregation from outside the stalked pole altered the viability of $C$. crescentus, we analyzed CFU of cells that had undergone ori/centromere translocation away from the stalked pole in the absence of replication. Cells PM500 (fluorescent label near ori) and PM109 (fluorescent label ParB-centromere) were spotted immediately after DnaA depletion time ( $1 \mathrm{~h}$ to $3 \mathrm{~h}$ ) on plates containing the inducer for $d n a A$ expression (supplemented with vanillate). Our CFU analyses revealed no significant differences between cells that initiated chromosome replication/segregation from the new pole (or mid-cell) compared to wild type conditions. Our data suggest that cells can recover relatively quickly after initiating chromosome replication/segregation from outside the stalked pole.

\section{DISCUSSION}

By inducing the movement of ori and centromere away from their intrinsic sub-cellular location in a replication-independent manner, we have shown that the molecular machineries involved in chromosome replication and segregation are remarkably flexible. Our data revealed that the activity of DnaA and ParA are not restricted to a single subcellular location inside the cell and can successfully induce chromosome replication and segregation from outside the stalked pole in C. crescentus. These results suggest that the set of proteins that regulate the asymmetric organization of chromosome replication and segregation must also rearrange, posing a model where these vital chromosomal loci (ori and centromere) play a key role in the localization of regulators that maintain cellular asymmetry. Cells that initiate chromosome replication and segregation from outside the stalked pole display no detectable physiological defects compared to wild type conditions. Thus, our data expose the remarkable ability of the cell to reorganize its chromosome along with the set of protein regulators involved in chromosomal maintenance in a relatively short time.

\section{DnaA's activity as replication initiator and cell asymmetry}

In the absence of membrane-bound organelles, bacteria rely on proteins organized in gradients to establish cellular polarity and carry out asymmetric functions. One example of such organization is the phosphorylated regulator CtrA (CtrA P), which binds ori and inhibits DnaA from initiating replication at one cell pole [37-39]. In pre-divisional cells, a phospho-signaling relay at the cell poles generates an asymmetric concentration gradient of CtrA P with the highest levels at the new pole that gradually decrease toward the stalked pole [40]. Alterations to the asymmetric concentration gradient of CtrA P eliminates the asymmetric regulation of DnaA, resulting in cells initiating replication from the new pole [40]. Our data revealed that DnaA can also trigger replication initiation from the new pole in cells with altered subcellular location of ori (Fig. 3). Our results can be explained by the undetectable levels in Western blot assays of CtrA in cells depleted of DnaA [41,42]. DnaA is a transcriptional regulator of $g c r A$, and GcrA is a transcriptional regulator of ctrA [41-43]. Consequently, the expression of ctrA is indirectly dependent on the levels of DnaA. Thus, cells with ori localized at the new pole with depleted 
levels of DnaA are likely to have none or minimal CtrA P gradient that is not sufficient to inhibit replication initiation from either pole.

\section{Regulation of periodicity of DnaA's activity}

Most of the regulators of DnaA's activity that have been identified so far are negative regulators that prevent the over-initiation of chromosome replication. However, positive regulators that trigger DnaA to initiate replication with such efficient periodicity remain limited. This periodicity of DnaA's activity is maintained even in E. coli cells that were artificially designed to have two spatially separated oris. In those cells, DnaA productively initiated replication synchronously from both oris [44]. In E. coli and $H$. pylori, a recruiter of DnaA has been characterized to promote the assembly of DnaA's polymer at ori [8-10]. Notably, constitutive expression of $d n a A$ has been shown to have no effect on the periodicity of DnaA's activity suggesting that $d n a A$ transcriptional regulation is not the principal modulator of DnaA's periodicity [18]. In C. crescentus, CtrA regulates the spatial activity of DnaA so that chromosome replication only initiates in the stalked cells [18, 38, 39]. However, CtrA is not involved in the periodic activity of DnaA [18]. Thus, the molecular mechanism that triggers DnaA to initiate chromosome replication with such precise periodicity remains unclear. A hypothetical scenario is that some type of regulator that facilitates this periodicity process is found within the microenvironment of where ori is localized at the time of replication initiation. Our data advocates that there is no regulator/modulator of DnaA that is fixed at the stalked pole of $C$. crescentus. We cannot however exclude the possibility that a potential modulator does exist, and this modulator could migrate along with ori because it either binds directly to ori or to DnaA. More work characterizing details about DnaA's activity at ori is required to identify the potential modulator of periodicity or the mechanism that DnaA uses to regulate its temporal activity with such remarkable accuracy.

\section{Localization of the centromere dictates the orientation of ParA's activity}

The partitioning protein ParA is another example of a protein in bacteria organized in gradients to establish cellular polarity. In C. crescentus, ParA forms a gradient with concentrations gradually decreasing from the new pole to the stalked pole [26-28]. Notably, this stable gradient of ParA is established well before chromosome replication and segregation are initiated [26-28]. Thus, the question remains as to what activates this asymmetric organization of ParA. Our data revealed that the organization of the ParA gradient can be completely reconstructed in the opposite orientation by rearranging the location of the centromere (Fig. 6A). We show that ParA can successfully segregate the centromere from the new pole to the stalked pole, which is the reverse direction to its wild type mechanism. We propose that once a stable gradient of ParA is formed in cells with translocated unreplicated-centromeres, the ParA-DNA interaction relay previously shown to provide the force necessary for centromere segregation [45] can initiate and segregate one centromere in the reverse direction.

\section{Robustness of cells to re-organize}

The view that bacteria are simply a bag of enzymes with no level of organization is long gone. Instead, the appreciation of the high levels of organization that bacteria manage in such limited spaces is mind blowing. In C. crescentus, an array of signaling proteins localize differentially at each pole. This organization orchestrates the progression of the cell cycle (i.e. chromosome replication, segregation, cytokinesis) with the development of the stalked and new pole. The centromere in $C$. crescentus serves as a hub of proteins involved in multiple important functions involved polar development. In this work, we asked what happens to the ability of cells to grow when the 
organization of the two key chromosomal loci (ori and centromere) are flipped in orientation. We first showed that the regulators (DnaA and ParA) can easily follow the new location of these sites and proceed with their activities, and in the case of ParA proceed segregation in the reverse direction. Notably, cells were able to recover the "forced" rearrangement of these chromosomal loci and continue to grow with no measurable delays. Our results revealed the robustness and flexibility that cells have to rearrange and reorganize the polar organization proteins that interact with ori and centromere.

\section{MATERIALS AND METHODS}

\section{Bacterial strains and growth conditions}

Wild-type strain CB15N (NA1000) was used to generate all C. crescentus derivatives (Table 1) in this study. Cells were grown in either rich (PYE) or minimal (M2G) medium [46] from freezer stock at 28 ${ }^{\circ} \mathrm{C}$. Growth medium was supplemented with vanillate (Sigma-Aldrich, $250 \mu \mathrm{M}$ ) and/or $0.3 \%$ D-(+)xylose (Sigma-Aldrich) as specified. When noted, the liquid/solid media was supplemented with appropriate antibiotics; for $C$. crescentus kanamycin $5 / 25 \mu \mathrm{g} / \mathrm{mL}$, streptomycin $5 / 5 \mu \mathrm{g} / \mathrm{mL}$, tetracycline $1 / 2 \mu \mathrm{g} / \mathrm{mL}$, and for $E$. coli kanamycin $30 / 50 \mathrm{mg} / \mathrm{mL}$.

Plasmids constructed in this study were created by cloning PCR products amplified using wild-type CB15N (NA1000) genomic DNA or Yersinia pestis KIM5 pMT1 DNA into pNPTS138, pXCHYC-2 and pXCFPN-2 [33] vectors. The constructs were transformed into $E$. coli DH5 $\alpha$ cells and grown at $37^{\circ} \mathrm{C}$ in Luria-Bertani (LB) medium. All primers used for cloning are listed in Table 2. Plasmid carrying CFPpMT1 parB was done by the pMT1 parB gene sequence isolation with $\mathrm{Kpnl}$ and Nhel restriction from PM396 (LS5269) and ligation to the equally treated xylose-inducible integrating plasmid pXCFPN-2 $\left(\mathrm{kan}^{\mathrm{R}}\right)$ [24]. parA gene was cloned into integrating $\mathrm{pXCHYC-2}\left(\mathrm{kan}^{\mathrm{R}}\right)$ plasmid under a xylose inducible promoter to express mCherry tagged C-terminal protein fusions [33]. Gibson cloning method [47] was used to construct the plasmids used to delete or insert a gene into the $C$. crescentus genome. To insert pMT1 parS site approximately $1 \mathrm{~kb}$ far away from the ori, the cloned pMT1 parS sequence from PM395 (LS5270) and around 600bp of CCNA0001 C-terminus and CCNA0002 N-terminus sequences were assembled into pNPTS138 plasmid [24]. The plasmid used for vanA::dnaA substitution was accomplished by assembling into pNPTS138 plasmid the cloned $d n a A$ gene sequence and $600 \mathrm{bp}$ of the flanking upstream and downstream sequences of vanA. For dnaA deletion purposes, $600 \mathrm{bp}$ of flanking upstream and downstream sequences of $d n a A$ were cloned into pNPTS138 plasmid.

\section{Growth assays}

Overnight cultures grown from $C$. crescentus frozen stocks in M2G liquid media were diluted to 0.2 $\mathrm{OD}_{600 \mathrm{~nm}}(2 \mathrm{~mL})$ in $13 \mathrm{~mm}$ glass tubes. Culture tubes were incubated at $28{ }^{\circ} \mathrm{C}$ and the optical density at $\mathrm{OD}_{600 \mathrm{~nm}}$ was monitored every hour to monitor the growth of bacteria. Rate of bacterial growth was calculated using the exponent of the growth curves on semi-log plots.

\section{Synchrony}

A culture of $C$. crescentus in M2G (15 mL) was inoculated with a saturated overnight M2G culture and was grown up to $\mathrm{OD}_{600}$ around 0.3 . Media was supplemented with vanillate $(250 \mu \mathrm{M})$ and antibiotics as noted. Cells were pelleted using centrifugation at $6000 \mathrm{rpm}$ for $10 \mathrm{~min}$ at $4{ }^{\circ} \mathrm{C}$. The cell pellet was resuspended in about $800 \mu \mathrm{L}$ of $1 \times \mathrm{x} 2$ salts and mixed well with percoll (900 $\mu \mathrm{L}$, Sigma Aldrich). Swarmer cells (bottom layer) were collected and separated out from the stalked/predivisional cells 
(top layer) using percoll density gradient by centrifuging at $11,000 \mathrm{rpm}$ for $20 \mathrm{~min}$ at $4{ }^{\circ} \mathrm{C}$. The cells were washed twice with cold 1x M2 salts by spinning at $8000 \mathrm{rpm}$ for $3 \mathrm{~min}$ at $4{ }^{\circ} \mathrm{C}$ and resuspended in $M 2 G$ media to the appropriate $\mathrm{OD}_{600}$. When cells were not synchronized, the cultures grown to around $0.3 \mathrm{OD}_{600}$ were pelleted and washed with $1 \times \mathrm{x} 2$ salts three times.

\section{Fluorescence microscopy}

The cells (1-3 $\mu \mathrm{L})$ were spotted on agar pads (1\% agarose in $\mathrm{M} 2 \mathrm{G})$ and imaged using phase contrast and fluorescent microscopy in Zeiss Axio Observer 2.1 inverted microscope, set up with a PlanApochromat 100x/1.40 Oil Ph3 M27 (WD=0.17 mm) objective, AxioCam 506 mono camera and ZEN lite software. Agar pads supplemented with vanillate $(250 \mu \mathrm{M})$ were used in time-lapse assays when needed. Images were analyzed using ImageJ software (https://imagej.nih.gov/ij/docs/menus/analyze.html) [48] and localization of fluorescent foci was counted using the Cell Counter plugin.

\section{Colony forming units (CFU) assay}

Serial dilutions of culture in ten-fold were done by mixing $10 \mu \mathrm{L}$ of culture with $90 \mu \mathrm{L}$ of PYE medium in a sterile 96 well plate. $5 \mu \mathrm{L}$ of each sample was spotted on PYE agar (1.5\%) plates supplemented with vanillate $(250 \mu \mathrm{M})$ if needed. CFU counts were obtained from the plates incubated at $28{ }^{\circ} \mathrm{C}$ for two days.

\section{AUTHOR CONTRIBUTIONS}

ABM, IPM, and PEM designed the study, acquired/analyzed data, and wrote the manuscript. 


\section{REFERENCES}

1. Hwang, D.S. and A. Kornberg, Opening of the replication origin of Escherichia coli by DnaA protein with protein HU or IHF. J Biol Chem, 1992. 267(32): p. 23083-23086.

2. Boye, E., A. Lobner-Olesen, and K. Skarstad, Limiting DNA replication to once and only once. EMBO Rep, 2000. 1(6): p. 479-83.

3. Sekimizu, K.B., D; Kornberg, A, Sequential early stages in the in vitro initiation of replication at the origin of the Escherichia coli chromosome. J Biol Chem, 1988. 263: p. 7124-7130.

4. Katayama, T., et al., Regulation of the replication cycle: conserved and diverse regulatory systems for DnaA and oriC. Nat Rev Microbiol, 2010. 8(3): p. 163-70.

5. $\quad$ Erzberger, J.P., M.L. Mott, and J.M. Berger, Structural basis for ATP-dependent DnaA assembly and replication-origin remodeling. Nat Struct Mol Biol, 2006. 13(8): p. 676-83.

6. Roth, A.M., W, High-anity binding sites for the initiator protein DnaA on the chromosome of Escherichia coli. Mol Microbiol, 1998. 28: p. 395-401.

7. Kitagawa, R.M., H; Okazaki, T; and Ogawa, T., A novel DnaA protein-binding site at $94.7 \mathrm{~min}$ on the Escherichia coli chromosome. Mol Microbiol, 1996. 19: p. 1137-1147.

8. Zawilak-Pawlik, A., et al., DiaA/HobA and DnaA: a pair of proteins co-evolved to cooperate during bacterial orisome assembly. J Mol Biol, 2011. 408(2): p. 238-51.

9. Ishida, T., et al., DiaA, a novel DnaA-binding protein, ensures the timely initiation of Escherichia coli chromosome replication. J Biol Chem, 2004. 279(44): p. 45546-55.

10. Zawilak-Pawlik, A., et al., HobA--a novel protein involved in initiation of chromosomal replication in Helicobacter pylori. Mol Microbiol, 2007. 65(4): p. 979-94.

11. McGarry KC, R.V., Grimwade JE, Leonard AC., Two discriminatory binding sites in the Escherichia coli replication origin are required for DNA strand opening by initiator DnaAATP. Proc. Natl. Acad. Sci., 2004. 101: p. 2811-2816.

12. Kato, J. and T. Katayama, Hda, a novel DnaA-related protein, regulates the replication cycle in Escherichia coli. Embo J, 2001. 20(15): p. 4253-62.

13. Zakrzewska-Czerwin'ska, J.J., D; Zawilak-Pawlik, A; Messer, Regulation of the initiation of chromosomal replication in bacteria. FEMS Microbiol. Rev., 2007. 31: p. 378-387.

14. Collier, J. and L. Shapiro, Feedback control of DnaA-mediated replication initiation by replisome-associated HdaA protein in Caulobacter. J Bacteriol, 2009. 191(18): p. 5706-16.

15. Li, Z., et al., Restoration of growth to acidic phospholipid-deficient cells by DnaA(L366K) is independent of its capacity for nucleotide binding and exchange and requires DnaA. J Biol Chem, 2005. 280(11): p. 9796-801.

16. Boeneman, K. and E. Crooke, Chromosomal replication and the cell membrane. Curr Opin Microbiol, 2005. 8(2): p. 143-8.

17. Collier, J., H.H. McAdams, and L. Shapiro, A DNA methylation ratchet governs progression through a bacterial cell cycle. Proc Natl Acad Sci U S A, 2007. 104(43): p. 17111-6.

18. Jonas, K., Y.E. Chen, and M.T. Laub, Modularity of the bacterial cell cycle enables independent spatial and temporal control of DNA replication. Curr Biol, 2011. 21(13): p. 1092-101.

19. Leslie, D.J., et al., Nutritional Control of DNA Replication Initiation through the Proteolysis and Regulated Translation of DnaA. PLoS Genet, 2015. 11(7): p. e1005342.

20. Schrader, J.M., et al., Dynamic translation regulation in Caulobacter cell cycle control. Proc Natl Acad Sci U S A, 2016. 113(44): p. E6859-E6867.

21. Bowman, G.R., et al., Caulobacter PopZ forms a polar subdomain dictating sequential changes in pole composition and function. Mol Microbiol, 2010. 
22. Bowman, G.R., et al., A polymeric protein anchors the chromosomal origin/ParB complex at a bacterial cell pole. Cell, 2008. 134(6): p. 945-55.

23. Ebersbach, G., et al., A self-associating protein critical for chromosome attachment, division, and polar organization in caulobacter. Cell, 2008. 134(6): p. 956-68.

24. Toro, E., et al., Caulobacter requires a dedicated mechanism to initiate chromosome segregation. Proc Natl Acad Sci U S A, 2008. 105(40): p. 15435-40.

25. Thanbichler, M. and L. Shapiro, MipZ, a spatial regulator coordinating chromosome segregation with cell division in Caulobacter. Cell, 2006. 126(1): p. 147-62.

26. Ptacin, J.L., et al., A spindle-like apparatus guides bacterial chromosome segregation. Nat Cell Biol, 2010. 12(8): p. 791-8.

27. Schofield, W.B., H.C. Lim, and C. Jacobs-Wagner, Cell cycle coordination and regulation of bacterial chromosome segregation dynamics by polarly localized proteins. EMBO J, 2010. 29(18): p. 3068-81.

28. Shebelut, C.W., et al., Caulobacter chromosome segregation is an ordered multistep process. Proc Natl Acad Sci U S A, 2010. 107(32): p. 14194-8.

29. Viollier, P.H., et al., Rapid and sequential movement of individual chromosomal loci to specific subcellular locations during bacterial DNA replication. Proc Natl Acad Sci U S A, 2004. 101(25): p. 9257-62.

30. Surovtsev, I.V., H.C. Lim, and C. Jacobs-Wagner, The Slow Mobility of the ParA Partitioning Protein Underlies Its Steady-State Patterning in Caulobacter. Biophys J, 2016. 110(12): p. 2790-2799.

31. Mera, P.E., V.S. Kalogeraki, and L. Shapiro, Replication initiator DnaA binds at the Caulobacter centromere and enables chromosome segregation. Proc Natl Acad Sci U S A, 2014. 111(45): p. 16100-5.

32. Lindler, L.E., et al., Complete DNA sequence and detailed analysis of the Yersinia pestis KIM5 plasmid encoding murine toxin and capsular antigen. Infect Immun, 1998. 66(12): p. 573142.

33. Thanbichler, M., A.A. Iniesta, and L. Shapiro, A comprehensive set of plasmids for vanillateand xylose-inducible gene expression in Caulobacter crescentus. Nucleic Acids Res, 2007. 35(20): p. e137.

34. Mohl, D.A. and J.W. Gober, Cell cycle-dependent polar localization of chromosome partitioning proteins in Caulobacter crescentus. Cell, 1997. 88(5): p. 675-84.

35. Easter, J., Jr. and J.W. Gober, ParB-stimulated nucleotide exchange regulates a switch in functionally distinct ParA activities. Mol Cell, 2002. 10(2): p. 427-34.

36. Ptacin, J.L., et al., Bacterial scaffold directs pole-specific centromere segregation. Proc Natl Acad Sci U S A, 2014. 111(19): p. E2046-55.

37. Laub, M.T., et al., Genes directly controlled by CtrA, a master regulator of the Caulobacter cell cycle. Proc Natl Acad Sci U S A, 2002. 99(7): p. 4632-7.

38. Quon, K.C., G.T. Marczynski, and L. Shapiro, Cell cycle control by an essential bacterial twocomponent signal transduction protein. Cell, 1996. 84(1): p. 83-93.

39. Quon, K.C., et al., Negative control of bacterial DNA replication by a cell cycle regulatory protein that binds at the chromosome origin. Proc. Natl. Acad. Sci. USA, 1998. 95(1): p. 1205.

40. Chen, Y.E., et al., Spatial gradient of protein phosphorylation underlies replicative asymmetry in a bacterium. Proc Natl Acad Sci U S A, 2011. 108(3): p. 1052-7.

41. Hottes, A.K., L. Shapiro, and H.H. McAdams, DnaA coordinates replication initiation and cell cycle transcription in Caulobacter crescentus. Mol Microbiol, 2005. 58(5): p. 1340-53.

42. Collier, J., S.R. Murray, and L. Shapiro, DnaA couples DNA replication and the expression of two cell cycle master regulators. EMBO J, 2006. 25(2): p. 346-56. 
43. Laub, M.T., et al., Global analysis of the genetic network controlling a bacterial cell cycle. Science, 2000. 290(5499): p. 2144-8.

44. Wang, X., et al., Replication and segregation of an Escherichia coli chromosome with two replication origins. Proc Natl Acad Sci U S A, 2011. 108(26): p. E243-50.

45. Lim, H.C., et al., Evidence for a DNA-relay mechanism in ParABS-mediated chromosome segregation. Elife, 2014. 3: p. e02758.

46. Ely, B., Genetics of Caulobacter crescentus. Methods Enzymol., 1991. 204: p. 372-84.

47. Gibson, D.G., et al., Enzymatic assembly of DNA molecules up to several hundred kilobases. Nat Methods, 2009. 6(5): p. 343-5.

48. Schneider, C.A., W.S. Rasband, and K.W. Eliceiri, NIH Image to ImageJ: 25 years of image analysis. Nat Methods, 2012.9(7): p. 671-5. 


\section{TABLES}

Table 1. List of strains and plasmids used in this study.

\begin{tabular}{|c|c|c|}
\hline $\begin{array}{l}\text { Strain or } \\
\text { plasmid }\end{array}$ & Description & $\begin{array}{l}\text { Source or } \\
\text { reference }\end{array}$ \\
\hline \multicolumn{3}{|l|}{$\begin{array}{l}\text { C. } \\
\text { crescentus } \\
\text { strains }\end{array}$} \\
\hline PM1 & Wild-type CB15N (NA1000) & $\begin{array}{l}\text { Evinger } \mathrm{M} \text { and } \\
\text { Agabian } \mathrm{N}, 1977\end{array}$ \\
\hline PM109 & $\begin{array}{l}\text { CB15N, } \Delta \text { vanA, parB::cfp-parB, dnaA:: } \Omega\left(\operatorname{strp}^{R} / \operatorname{spec}^{R}\right), \\
\text { vanA::dnaA }\end{array}$ & Mera et al, 2014 \\
\hline PM121 & $x y I X:: p a r A(D 44 A)-y f p\left(\operatorname{tet}^{R}\right)$ in PM109 $\left(\operatorname{strp}^{R}\right)$ & This study \\
\hline PM247 & $x y \mid X:: m$ Cherry-PopZ $\left(\right.$ tet $\left.^{R}\right)$ in PM109 $\left(\operatorname{strp}^{R}\right)$ & Mera et al, 2014 \\
\hline PM433 & CB15N, pMT1 parS & This study \\
\hline PM438 & CB15N, pMT1 parS, xylX::yfp-parB (pMT1) $\left(\mathrm{kan}^{\mathrm{R}}\right)$ & This study \\
\hline PM500 & $\begin{array}{l}\text { CB15N parS (pMT1), vanA::dnaA, } \Delta d n a A, x y I X:: c f p-p a r B \\
\text { (pMT1) }\left(\operatorname{kan}^{R}\right)\end{array}$ & This study \\
\hline PM503 & $\begin{array}{l}\text { pDNA219 (parA-mCherry under xylose promoter })\left(\operatorname{kan}^{R}\right) \text { in } \\
\text { PM109 }\left(\text { strp }^{R}\right)\end{array}$ & This study \\
\hline \multicolumn{3}{|l|}{ Plasmids } \\
\hline pNPTS138 & Nonreplicating vector for allelic replacement $\left(\mathrm{kan}^{\mathrm{R}}\right)$ oriT sacB & $\begin{array}{l}\text { Alley M. R. K., } \\
\text { unpublished data }\end{array}$ \\
\hline pXCHYC-2 & $\begin{array}{l}\text { Integrating plasmid for sylose inducible expression of } \\
\text { mCherry tagged CCNA_03869 }\left(\text { parA); }\left(\operatorname{kan}^{R}\right)\right.\end{array}$ & $\begin{array}{l}\text { Thanbichler M. et al, } \\
2007\end{array}$ \\
\hline pXCFPN-2 & $\begin{array}{l}\text { Integrating plasmid for xylose inducible expression of CFP } \\
\text { tagged parB(pMT1) }\left(\operatorname{kan}^{R}\right)\end{array}$ & $\begin{array}{l}\text { Thanbichler M. et al, } \\
2007\end{array}$ \\
\hline pDNA214 & $\begin{array}{l}\text { pNPTS138- parS (pMT1) sequence flanked by } 600 \mathrm{bp} \\
\text { UP/DWN at base } 1108 \text { for insertion of parS (pMT1) near ori. }\end{array}$ & This study \\
\hline pDNA216 & pXCFPN-2 - xyIX::cfp-parB (pMT1) $\left(\mathrm{kan}^{\mathrm{R}}\right)$ & This study \\
\hline pDNA217 & $\begin{array}{l}\text { pNPTS138- } d n a A \text { sequence flanked by } 600 \mathrm{bp} \text { UP/DWN of } \\
\text { CCNA_02476. To replace vanA with } d n a A\end{array}$ & This study \\
\hline pDNA218 & pNPTS138- 600bp UP/DWN CCNA_00008 for dnaA deletion & This study \\
\hline pJP49 & pXYFPC5-parA(D44A) & Ptacin J.L. et al, 2010 \\
\hline pDNA219 & $\begin{array}{l}\text { CCNA_03869 cloned into pXCHYC-2 at Ndel 5' and Sacl 3' } \\
\text { restriction enzyme sites }\end{array}$ & This study \\
\hline pMS138 & pMCS4 - parS (pMT1) & $\begin{array}{l}\text { Schwartz M.\& Shapiro } \\
\text { L., } 2011\end{array}$ \\
\hline pMS139 & pXYFPN-2 - xylX ::yfp-parB( pMT1) $\left(\mathrm{kan}^{\mathrm{R}}\right)$ & $\begin{array}{l}\text { Schwartz M. \& } \\
\text { Shapiro L., } 2011\end{array}$ \\
\hline
\end{tabular}


Table 2. List of primers used in this study. Primers that start with "Gib" were used in Gibson cloning technique [47].

\begin{tabular}{|l|l|}
\hline Primer name & \multicolumn{1}{|c|}{ 5’ to 3' sequence } \\
\hline Gib_UP600dnaA_fow & AGCTTCTCTGCAGGATATCTGGATCCGCGCGTGCGCACCTTC \\
\hline Gib_UP600dnaA_rev & TCTTCAATCCTACGATACGGTTTCGTCGTCCACCGCCTTGCA \\
\hline Gib_DWN600dnaA_fow & TGTTTTTGTGCAAGGCGGTGGACGACGAAACCGTATCGTAGG \\
\hline Gib_DWN600dnaA_rev & GCCGAAGCTAGCGAATTCGTGGATCTCGAAGCGGTGCTAGCG \\
\hline Gib_UP600bpVanA_fow & AGCTTCTCTGCAGGATATCTGGATCGGGCGCTCTCGACAGCG \\
\hline Gib_UP600vanA_rev & TGGCAACCCCGCCCTTCATGGTCATCGTCGTTTCCTCGCATC \\
\hline Gib_dnaA_fow & CCGAACCACGATGCGAGGAAACGACGATGACCATGAAGGGCGG \\
\hline Gib_dnaA_rev & GCGCGGGACGCCACCCGAACCTTGATTAGCCCCGCAGCTTGC \\
\hline Gib_DWN600vanA_fow & CCTGACGCGCAAGCTGCGGGGCTAATCAAGGTTCGGGTGGCG \\
\hline Gib_DWN600vanA_rev & GCCGAAGCTAGCGAATTCGTGGATCCGTCGATGACGTGGGTC \\
\hline Gib_CCNA_parSpMT1Up-fwd & GCTTCTCTGCAGGATATCTGGATCGGCTGCCGCAAGCTGGA \\
\hline S_Gib_CCNA_parSpMT1Up-rev & TGAAATCACCACGCTTTTCAACCTCTCATCCCAGGACCTCGA \\
\hline S_Gib_ParSpMT1_fow & GGCAAGGTCGAGGTCCTGGGATGAGAGGTTGAAAAGCGTGG \\
\hline S_Gib_ParSpMT1_rev & CGAGCGTAACAGGGGTGAGGCTCATAAGCTTTGTTTTTCACC \\
\hline S_Gib_CCNA_parSpMT1DWN_fwd & GAAATTGGCGTGGTGAAAAACAAAGCTTATGAGCCTCACCCCTG \\
\hline Gib_CCNA_parSpMT1DWN_rev & CGAAGCTAGCGAATTCGTGGATCTCATACGGTCAGGGCTC \\
\hline CCNA_ParA atg_5'Ndhel_fow & AAACATATGTCCGCTAATCCTCTCCG \\
\hline CCNA_ParA -3'-Sacl_rev & AAAGAGCTCtGGCGGCCTTGGCCTG \\
\hline
\end{tabular}




\section{FIGURE LEGENDS}

Figure 1. Cell-cycle dependent dynamics of Caulobacter crescentus. Localization of two chromosomal foci [origin of replication (ori) and centromere (parS)] and two proteins involved in chromosome segregation [ParA and PopZ] over the course of a normal cell cycle. Non-dividing cells have ori (cyan) and parS (green) localized near the stalked pole. PopZ anchors the centromere region parS and the parS-binding protein ParB complex at the stalked pole. Once replication initiates, two foci of ori and two foci of parS are observed. Upon the onset of chromosome replication and segregation, a second PopZ foci appears at the new pole. The new duplicated ori and parS are the first regions to move in a ParA-dependent manner to the new pole.

Figure 2. Translocation of ori triggered by DnaA in the absence of chromosome replication. (A) Time lapse of indicator strain [PM500: parS(pMT1), vanA::dnaA, $\triangle d n a A$, pxylX::cfp- parB(pMT1)] with fluorescent tag near ori $(\sim 1 \mathrm{~kb})$ and DnaA expression regulated by the vanillate promoter $P_{\text {VanA }}$. Cells grown in $M 2 G$ with vanillate were synchronized and swarmer cells were spotted on $1 \%$ agarose $M 2 G$ pads in the presence (top row) or absence (bottom row) of vanillate (250uM). Cells were imaged with phase contrast and CFP-mediated fluorescence microscopy every 30 minutes. (B) Plotted are the mean \pm SD percentages from three independent experiments of cells with translocated ori to the middle or new pole as time of DnaA depletion increases.

Figure 3. Chromosome replication is not limited to the stalked pole. A) Cells with fluorescent tag near ori [PM500, parS(pMT1) near ori, vanA::dnaA, $\Delta d n a A$, pxylX::cfp-parB (pMT1)] were synchronized and DnaA was depleted by growing them in liquid $M 2 G$ media without vanillate. At $3 \mathrm{~h}$ of DnaA depletion, un-replicated ori foci translocated to the opposite pole, middle or stayed at the stalked pole (left panel). After the depletion period, DnaA expression was induced by supplementation of vanillate $(250 \mu \mathrm{M})$. Within 30 minutes of DnaA repletion, cells were able to initiate chromosome replication (right panel) as evidenced by two ori (cyan) foci. B) The appearance of two replicated ori foci were quantified from three independent fluorescence microscopy time-lapses. The plot represents the mean \pm SD percent of cells that started replication at different time points. Analyses of two-way ANOVA in-between the frequencies of replication at stalk pole vs. new pole are significantly different at time points 30 -min and $45-\mathrm{min} ;{ }^{* * *} \mathrm{p}<0.001$ and ${ }^{*} \mathrm{p}<0.05$.

Figure 4. Translocated centromeres effectively segregate in opposite direction. (A) Time-lapse of centromere segregation (green foci represent CFP-ParB/parS) starting from the stalked pole, mid-cell or new pole of PM109 (parB::cfp-parB, dnaA::, vanA::dnaA) cells after $3 \mathrm{~h}$ of DnaA depletion. Cells imaged were synchronized prior to DnaA depletion in $\mathrm{M} 2 \mathrm{G}$ media $\left(2 \mathrm{~mL}, \mathrm{OD}_{600} \sim 0.1\right)$ and vanillate $(250 \mu \mathrm{M}$ ) was added (time $0 \mathrm{~min}$ ) to induce the expression of DnaA. $2 \mu \mathrm{L}$ of cells were spotted on $1 \%$ agarose pads supplemented with vanillate $(250 \mu \mathrm{M})$. Scale bar $=1 \mu \mathrm{m}$. (B) Chromosome segregation of synchronized PM109 (parB::cfp-parB, dnaA:: $\Omega$, vanA::dnaA) cells under wild-type conditions (media supplemented with vanillate). (C) Plotted are the mean \pm SD of cells with centromeres segregation to the cell poles based on initial localization of CFP-ParB/parS. Data represents three independent experiments. Statistical analyses of two-way ANOVA in-between the frequencies of segregation at mid-cell and new pole are significantly different at 45 -min and 60-min time points, ${ }^{* *} p<0.01$ and the frequencies of segregation at or near stalked pole and mid-cell at $45-\mathrm{min} .{ }^{*} p<0.05$.

Figure 5. Centromere segregation in the opposite direction requires active ParA. Cells with background parB::cfp-parB, dnaA:: $\Omega$, vanA::dnaA with either ParA ${ }^{\mathrm{WT}}$ (PM109) or ParA ${ }^{\mathrm{D} 44 \mathrm{~A}}$ (PM121) were grown in the absence of vanillate to allow for centromere translocation. After $3 \mathrm{~h}$ of DnaA depletion, cultures were supplemented with vanillate $(250 \mu \mathrm{M})$ to express DnaA and xylose $(0.3 \%)$ to induce the expression of ParA ${ }^{\mathrm{D} 44 \mathrm{~A}}$ variant protein. Cells were imaged by spotting $2 \mu \mathrm{L}$ of cells on $1 \%$ agarose pads. (A) Phase contrast fluorescence micrographs of PM109 expressing wild-type ParA ${ }^{\text {WT }}$ (left) and PM121 expressing ATP hydrolysis variant ParA ${ }^{\mathrm{DA4A}}$ (right). Scale bar $=1 \mu \mathrm{m}$. (B) Frequencies of centromere segregation were quantified based on localization of CFP-ParB (green 
foci). The data represents analyses of three independent experiments. Bar graph illustrates the mean $\pm \mathrm{SD}$ values. Statistical analysis: two-way ANOVA, ${ }^{* * *} \mathrm{P}<0.001$.

Figure 6. Localization of ParA and PopZ in cells with translocated centromeres. (A) Translocation of centromere to the new pole in DnaA depleted cells results in flipped ParA-mCherry gradient (red). Green foci represent CFP-ParB (centromeres). White arrows indicate location of the stalks. PM503 cells (parB::cfp-parB, dnaA:: $\Omega$, vanA::dnaA xyIX::parA-mCherry) were synchronized and depleted of DnaA for $3 \mathrm{~h}$. The culture was supplemented with xylose $(0.1 \%)$ for 2 hours during the time of depletion. After DnaA depletion ( $3 \mathrm{~h}$ ), $2 \mu \mathrm{L}$ of cells were mounted on $1 \%$ agarose pad and imaged using phase contrast fluorescence microscopy. Scale bar $=1 \mu \mathrm{m}$. (B \& C) Quantification of localization of PopZ in cells depleted (B) and repleted $(C)$ of DnaA. PM247 (parB::cfp-parB, dnaA:: $\Omega$, vanA::dnaA xyIX::mCherry-popZ) were grown in the absence of vanillate (DnaA depletion) for $3 \mathrm{~h}$ and then supplemented with vanillate (DnaA repletion) for $1 \mathrm{~h}$. Cultures were supplemented with xylose $(0.1 \%)$ to induce the expression of mCherry-PopZ for $1 \mathrm{~h}$ prior to isolation of swarmer cells. Phase contrast fluorescent micrographs were obtained just before and after 1 hour of the addition of vanillate. The data represents analyses of three independent experiments. Bar graph illustrates the mean $\pm S D$ values.

Figure 7. Depletion of DnaA for $3 \mathrm{~h}$ does not alter the viability of $C$. crescentus. Figure illustrates colony forming unit (CFU) assays of (A) parS (pMT1) vanA::dnaA, $\triangle d n a A, x y I X:: c f p-p a r B$ (pMT1; PM500) and (B) parB::cfp-parB, dnaA:: $\Omega$, vanA::dnaA (PM109) cells. The cultures $(3 \mathrm{~mL})$ grown up to $\mathrm{OD}_{600} \sim 0.3$ were washed three times with $1 \mathrm{X} \mathrm{M} 2$ salts as described under methods section and the $\mathrm{OD}_{600}$ were set to $\sim 0.2$ in M2G media $(2 \mathrm{~mL})$. DnaA was depleted for $1 \mathrm{~h}, 2 \mathrm{~h}$ and $3 \mathrm{~h}$ in separate cultures at $28^{\circ} \mathrm{C}$ and CFU assay were performed. Control sample (C) were not depleted of DnaA and incubated with vanillate $(250 \mu \mathrm{M})$ for $3 \mathrm{~h}$. PYE plates were incubated at $28^{\circ} \mathrm{C}$ for 2 days prior to obtaining the images. The data shown are a representative of three independent replicates. 
bioRxiv preprint doi: https://doi.org/10.1101/521781; this version posted January 16,2019 . The copyright holder for this preprint (which was not certified by peer review) is the author/funder, who has granted bioRxiv a license to display the preprint in perpetuity. It is made available under aCC-BY-NC-ND 4.0 International license.

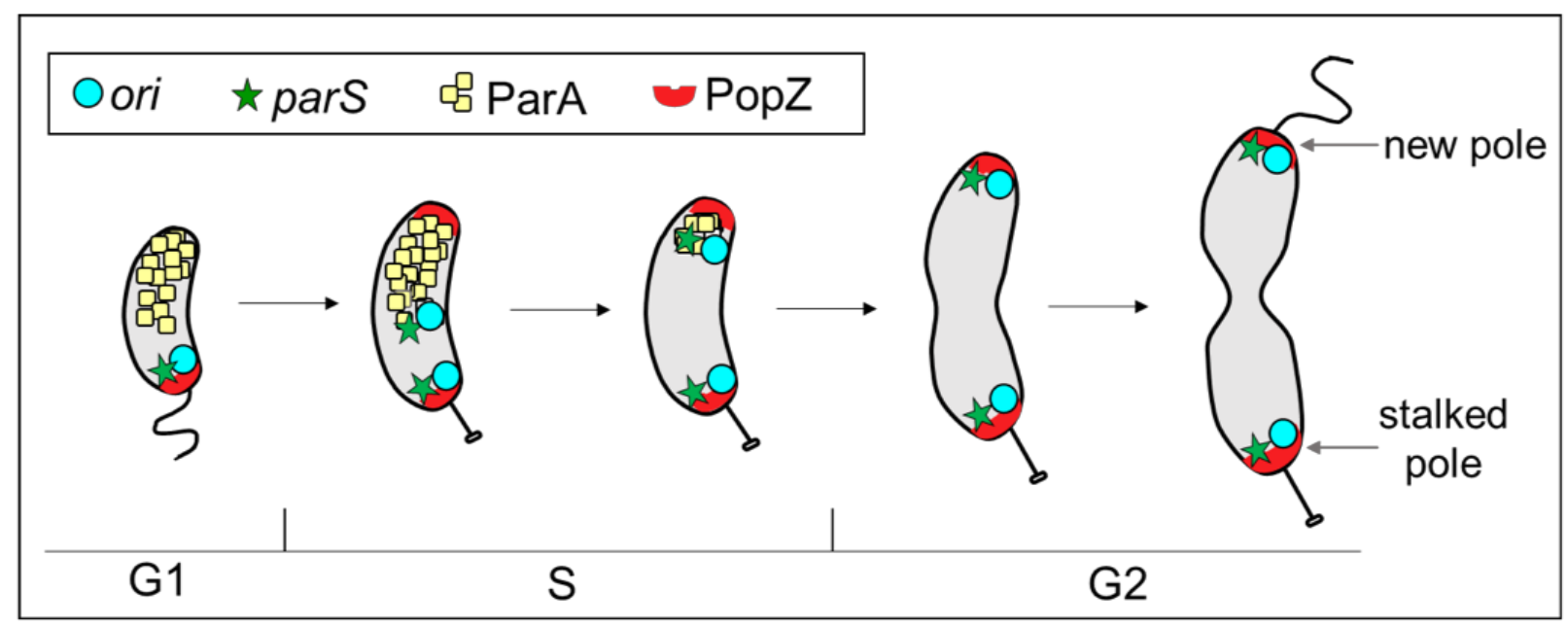

Figure 1 
bioRxiv preprint doi: https://doi.org/10.1101/521781; this version posted January 16,2019 . The copyright holder for this preprint (which was not certified by peer review) is the author/funder, who has granted bioRxiv a license to display the preprint in perpetuity. It is made available under aCC-BY-NC-ND 4.0 International license.

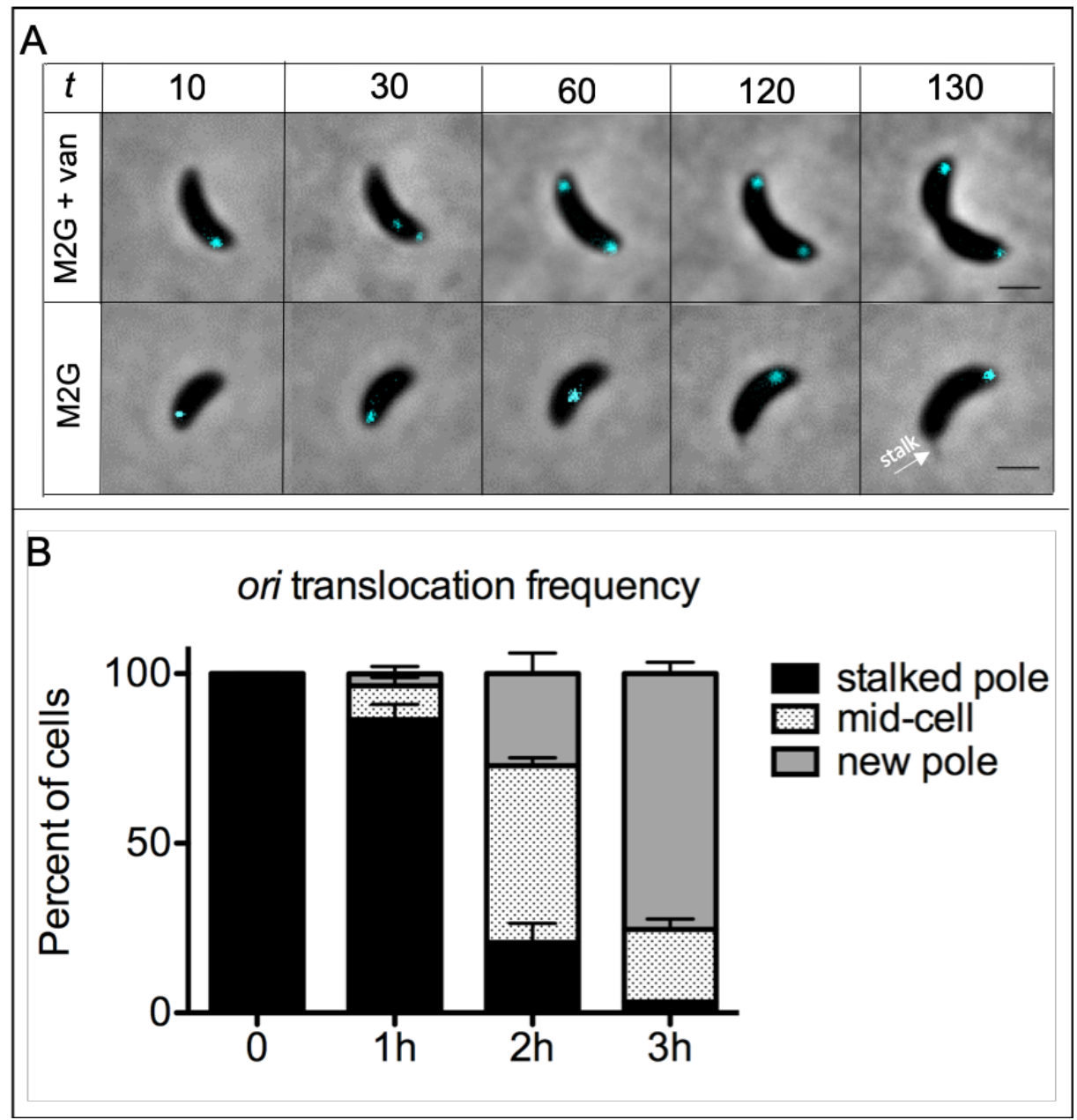

Figure 2 
bioRxiv preprint doi: https://doi.org/10.1101/521781; this version posted January 16,2019 . The copyright holder for this preprint (which was not certified by peer review) is the author/funder, who has granted bioRxiv a license to display the preprint in perpetuity. It is made available under aCC-BY-NC-ND 4.0 International license.

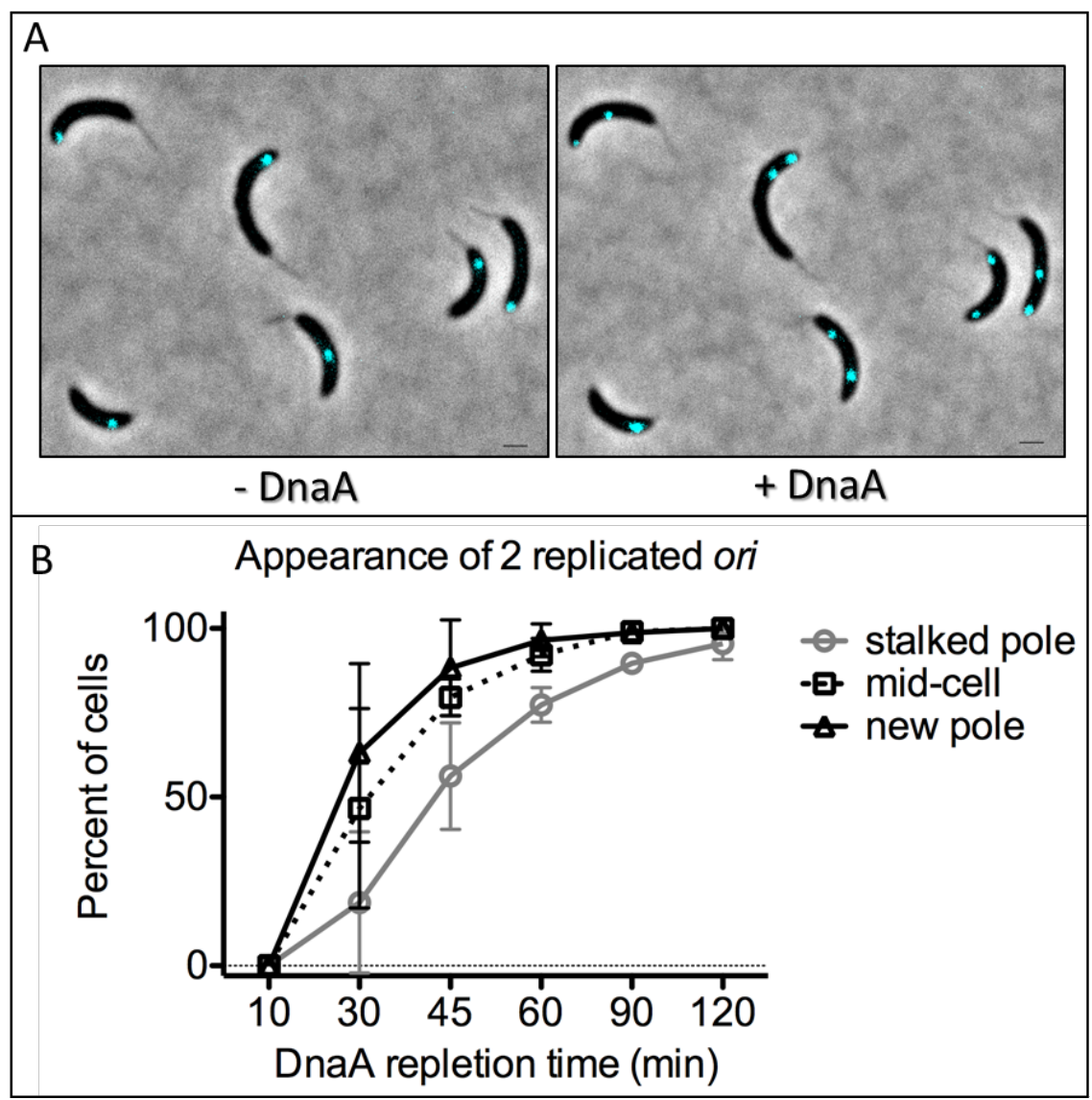

Figure 3 


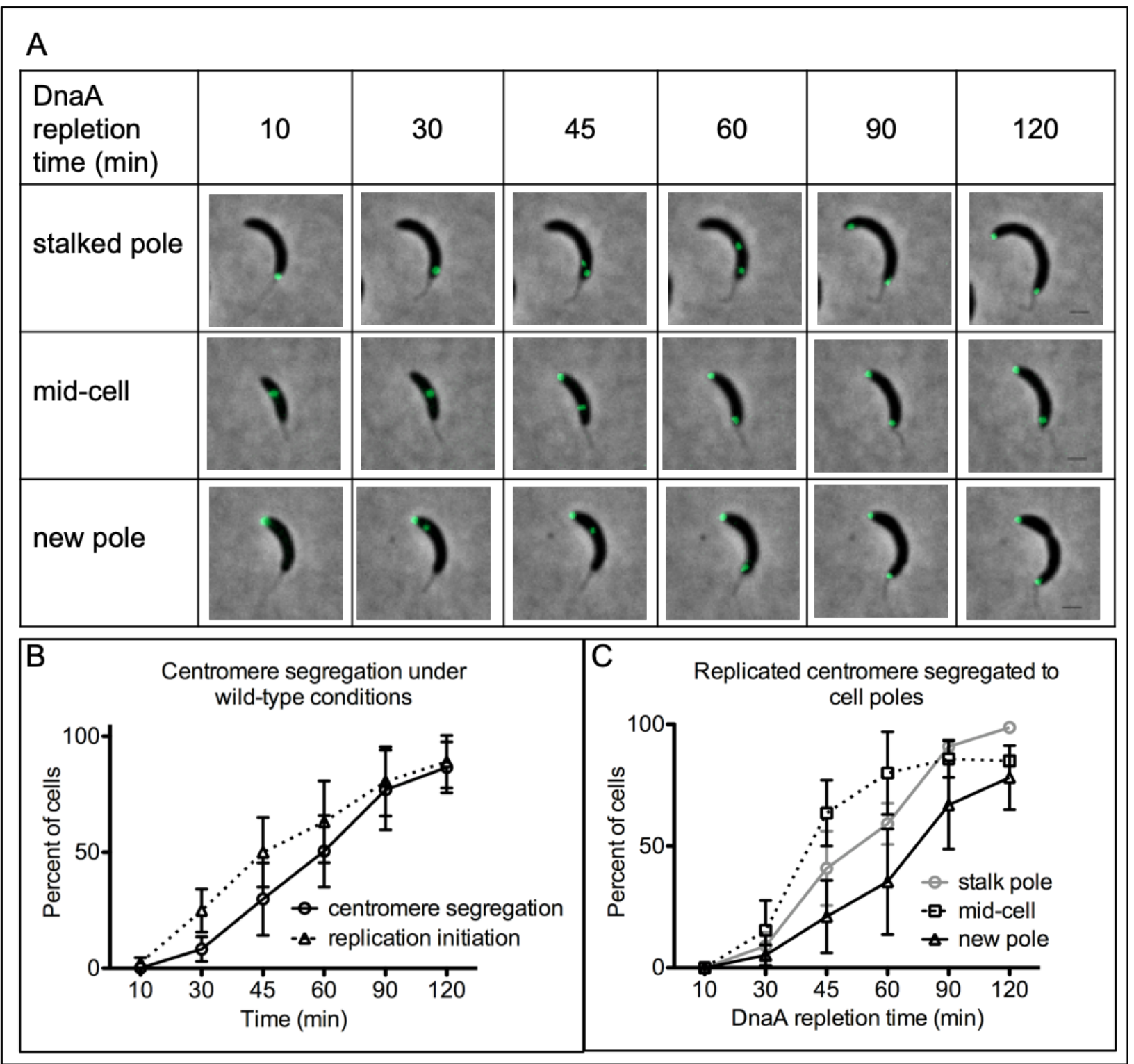

Figure 4 
bioRxiv preprint doi: https://doi.org/10.1101/521781; this version posted January 16,2019 . The copyright holder for this preprint (which was not certified by peer review) is the author/funder, who has granted bioRxiv a license to display the preprint in perpetuity. It is made available under aCC-BY-NC-ND 4.0 International license.

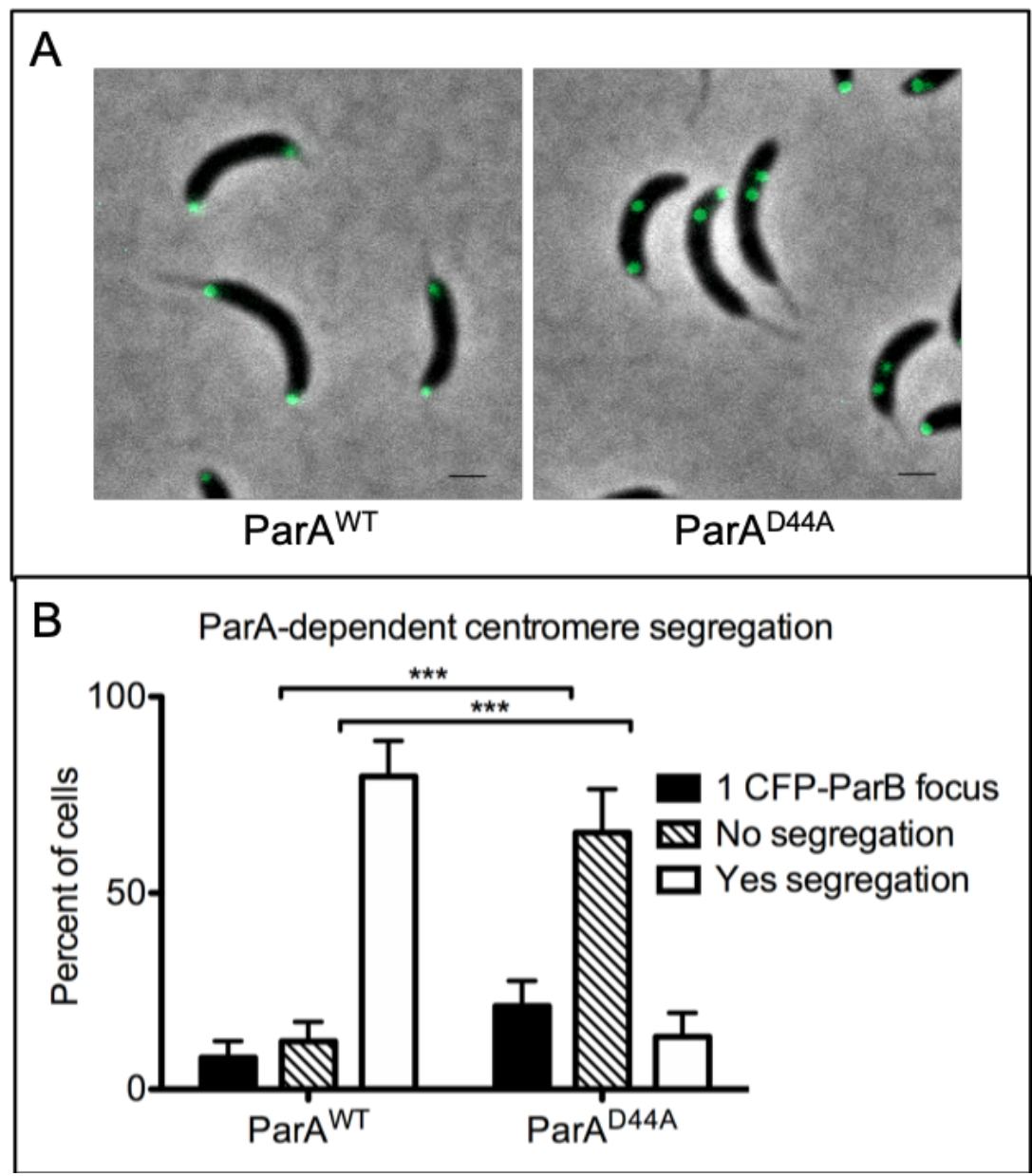

Figure 5 
bioRxiv preprint doi: https://doi.org/10.1101/521781; this version posted January 16,2019 . The copyright holder for this preprint (which was not certified by peer review) is the author/funder, who has granted bioRxiv a license to display the preprint in perpetuity. It is made available under aCC-BY-NC-ND 4.0 International license.

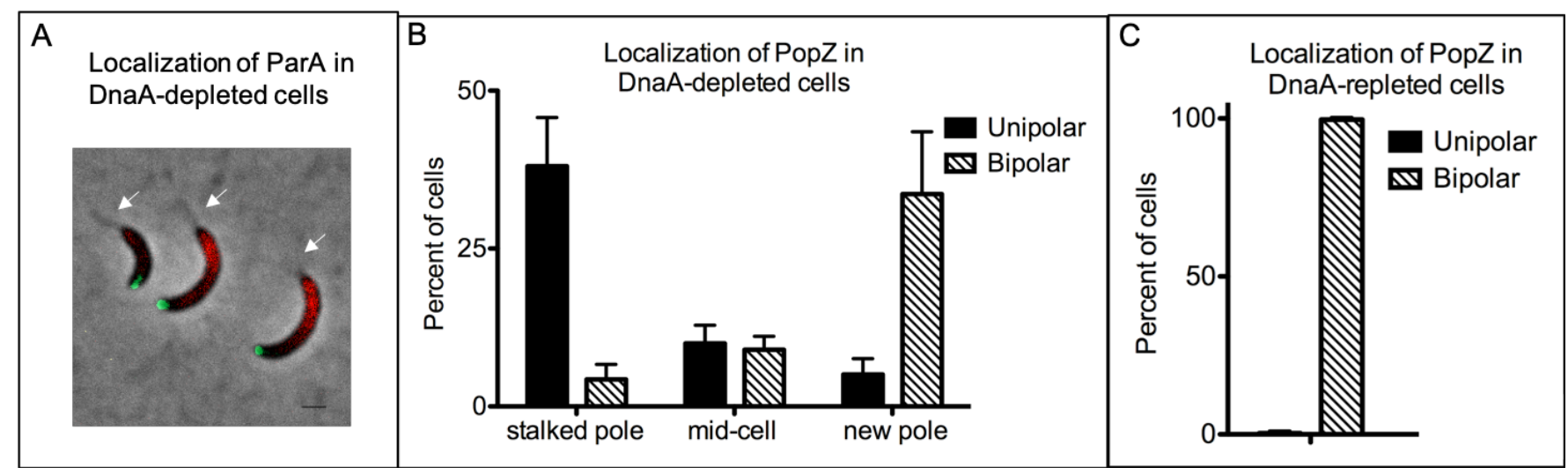

Figure 6 
bioRxiv preprint doi: https://doi.org/10.1101/521781; this version posted January 16,2019 . The copyright holder for this preprint (which was not certified by peer review) is the author/funder, who has granted bioRxiv a license to display the preprint in perpetuity. It is made available under aCC-BY-NC-ND 4.0 International license.

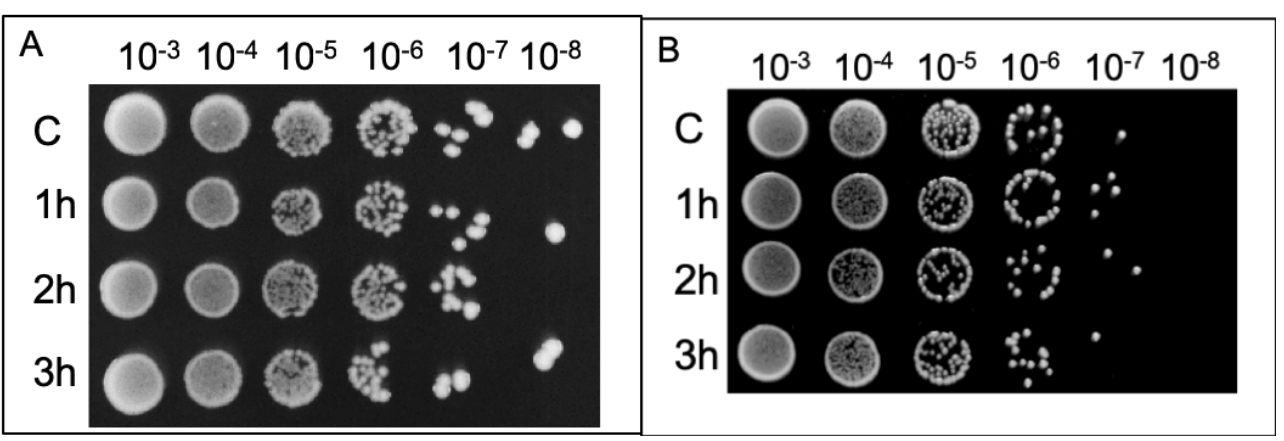

Figure 7 